\title{
Are COVID-19 test results masking important issues?
}

\author{
Brandon Owen ${ }^{1}$ and Simon Shepherd ${ }^{2}$
}

\section{A commentary on}

\section{Herron J B, Dennis J, Brennan PA.}

Coronavirus antibody positive tests and continued use of personal protective equipment throughout the pandemic. Br J Oral Maxillofacial Surg 2020; DOI: 10.1016/j.bjoms.2020.06.021.

\begin{abstract}
Basic health and infection control measures are the main methods of protection against COVID-19. Patients are well informed about how practitioners should be conducting themselves, however, they may lose trust in clinicians who fail to demonstrate, and promote those same basic prevention measures. The broader COVID-19 strategy has included the rapid development and deployment of swabs and antibody tests. Flaws in testing fail to offer assurances due to false negatives while even true positives cannot guarantee future immunity as there is uncertainty regarding long-term antibody response. An understanding of human factors and an appreciation of the limitations of available tests could offer healthcare staff mechanisms to encourage safety.
\end{abstract}

\section{Commentary}

Coronavirus (SARS-CoV-2) known as COVID-19 presents an unprecedented situation for public health and healthcare. The paper by Herron et al. presents a short, simplistic, diverse overview article exploring current information and issues being brought to our attention by COVID-19.

The authors explain how COVID-19 era relaxing of regulation has potentially resulted in products entering the market which have not undergone the same rigorous quality assurances normally expected. This highlights the need for practitioners to take a judicious approach when buying into new technology or services related to COVID-19 due to changing guidance, legislation and rapidly emerging evidence. As stated by a Department of Health and Social Care policy paper, 'An unreliable test is worse than no test'. ${ }^{1}$ Perhaps it is also true that a misinterpreted test is worse than no test?

The COVID-19 reverse transcription polymerase chain reaction (RT-PCR) swab and a COVID-19 antibody test have been hurriedly developed and deployed. The authors highlight a reported 30\% false negative rate for the COVID-19 RT-PCR swab. A swab result

\section{GRADE rating}

\section{Practice point}

- There is a limited evidence base regarding COVID-19 advancements. Health professionals must interpret emerging evidence carefully.

- A confirmed COVID-19 infection or positive antibody test is not a guarantee of future immunity. Appropriate personal protective equipment must be used at all times to protect patients and colleagues.

- Patients are well informed regarding how healthcare professionals should be conducting themselves. The patient-practitioner relationship may be harmed if patients perceive practitioners to not be following current guidance with respect to personal protective equipment, hand hygiene and social distancing.

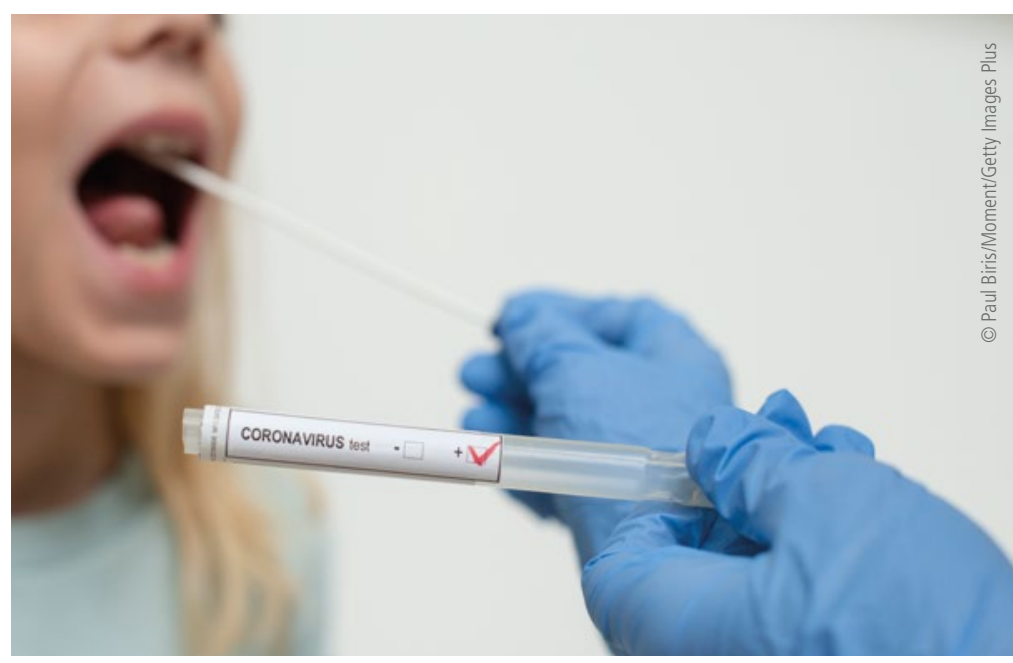

should not be used as the sole criteria when making decisions about patient care and should be interpreted along with clinical features. $^{2}$

COVID-19 antibody tests should provide a more reliable method of determining those who have been infected, a potentially important finding considering that in some individuals the infection is largely asymptomatic. ${ }^{3}$ However, it should be kept in mind that an antibody test is only an indication of past infection and not immunity. The authors cite limitations with current evidence regarding antibody response in studies with low numbers of participants and short follow up. One study is described where $30 \%$ of patients demonstrated very low neutralising antibody titre with $6 \%$ of these having no response at all after 2 weeks. There is therefore a potential risk that individuals may lack sufficient immune response with risk of becoming re-infected.

Plans to combat the COVID-19 crisis have included the development of a group of medical personnel who are immune as a means of reducing pressures on personal protective equipment (PPE) resources. Given the uncertainty regarding swabs, antibody test flaws and ambiguity over long-term immunity following infection, the authors quite rightly state that basic health 
measures will continue to be crucial for preventing COVID-19 transmission.

As new evidence emerges, we may be able to determine the risk of re-infection, length of the antibody response, and the need to re-test individuals to monitor this. ${ }^{4}$ It is important practitioners do not misinterpret a positive COVID-19 antibody test as offering a degree of future immunity.

The authors focus the second half of their article on mask wearing. They stress that it is imperative that all healthcare staff continue to wear masks irrespective of whether they have been infected with COVID-19. The authors raise an important point that patients may lose trust in the health professional who does not wear a mask as they may be viewed as disregarding the rules. This is important considering the compulsory use of facemasks and coverings within hospitals, public transport and shops. It seems sensible that all aspects of PPE and social distancing must be maintained in the presence of patients where possible. Patients are perhaps more informed now than they have ever been regarding how health professionals should be conducting themselves. The authors conclude that healthcare professionals should be mindful of their actions, responsibilities and the need to provide adequate role modelling for colleagues. Some patients will be anxious about attending the dental setting so it is imperative that staff and the environment they manufacture encourages patient confidence.

A theme within the article is that of human factors. The authors discuss human factor strategies such as team briefings, flattening hierarchy and encouraging assertive followership. The authors state that human factor strategies must be examined to reduce risk associated with lack of engagement from healthcare staff and patients. Human factors appreciate human characteristics and design work systems to support performance and safety. ${ }^{5}$ Many staff reportedly remain unaware of the importance of human factors. Simply raising awareness may be ineffective; it is the design of the system that should be modified to better aid staff and address problems. ${ }^{5}$ This includes interventions from national policy level to the individual level.

One potential limitation of this paper is its diverse nature touching superficially on multiple themes. Considering the wealth of global COVID-19 literature this article does, however, serve as an important reminder to practitioners of new advancements. However, these new advancements may come with unrefined or tentative outcomes. Basic health and infection control measures will therefore remain the pillar of protecting patients, staff and the public until advancements such as a vaccine can offer additional protection.

\section{Author affiliations}

${ }^{1}$ Oral Surgery Dental Core Trainee, Dundee Dental Hospital, Dundee, Scotland; ${ }^{2}$ Locum Consultant Oral Surgery, Dundee Dental Hospital, Dundee, Scotland

\section{References}

1. Department of Health and Social Care. Coronavirus (COVID-19) Scaling up our testing programmes. Available at https://www.gov.uk/government/publications/ coronavirus-covid-19-scaling-up-testing-programmes (accessed August 2020).

2. Tahamtan A, Ardebili A. Real-time RT-PCR in COVID-19 detection: issues affecting the results. Expert Rev Mol Diagn 2020; DOI: 10.1080/14737159.2020.1757437.

3. Arons M M, Hatfield K M, Reddy S C et al. Presymptomatic SARS-CoV-2 infection and transmission in a skilled nursing facility. New Eng J Med 2020; DOI: 10.1056/ NEJMoa2008457.

4. Burki T K. Testing for COVID-19. Lancet Respir Med 2020; DOI: 10.1016/ S2213-2600(20)30247-2.

5. Russ A L, Fairbanks R J, Karsh B T, Militello L G, Saleem J J, Wears R L. The science of human factors: separating fact from fiction. BMJ Qual Saf 2013; 22: 802-808.

Evidence-Based Dentistry (2020) 21, 82-83.

https://doi.org/ 10.1038/s41432-020-0117-3 